a different mechanism based on observations of chromosome aberrations in Tradescantia microspores irradiated with very soft X-rays. Neary's theory is that production of a chromosome aberration results from interaction between two damaged targets, in each of which the damage is produced by a single energy-loss event. The increased effectiveness of densely ionizing radiation is due to the greater chance of interaction between the two damaged targets when they are produced close together by a single particle. Neary also suggests that cellular killing by radiation is due to the same mechanism. This theory gave results agreeing well with the observations when irradiations were made in aerobic conditions. Under anoxic conditions, however, the RBE was found to rise too quickly with increasing density of ionization. This observation could be explained only by invoking a further hypothesis, namely, that oxygen was actually produced in the tracks of densely ionizing radiation, a hypothesis first made explicitly by Shchepotieva, Ardashnikov, Lurje and Rahkmanova (Izvestia Akademii Nauk USSR, biological series No. 4,642,1961) and suggested by many previous observations that oxygen is produced when pure water is irradiated by $\alpha$ particles but not by $\mathrm{X}$-rays.

Recently, Alper and her colleagues have been able to test this hypothesis experimentally (Brit. J. Radiol., 40, 843; 1967, and Radiation Research, 32, 780; 1967). The rests were based on irradiation of bacteria with X-rays and fast neutrons, the latter producing recoil protons with fairly dense ionization. The sensitivity of the bacteria was studied as a function of oxygen concentration using both types of radiation. The results fitted the model of oxygen production by densely ionizing radiation and the concentration of oxygen formed in the secondary tracks from fast neutrons was found to be $0.83 \mu$ mole per litre. An independent test was also made by comparing the relative sensitivity in the presence and absence of oxygen (OER) of several strains of bacteria irradiated with X-rays and fast neutrons. A theoretical interpretation of the observed relationship between the values of OER with X-rays and neutrons led to a value for the oxygen produced, in the densely ionized tracks, of $0.76 \mu$ mole per litre. The agreement with the first model is remarkable considering the experimental uncertainties. This confirmation of the importance of oxygen production in the tracks of densely ionizing particles is a valuable support for Neary's theory.

\section{Cysteamine as a Radioprotector}

\section{from our Medical Biochemistry Correspondent}

IT is well known that cysteamine (the amine formed by decarboxylation of the amino-acid cysteine) can protect cells against the effects of ionizing radiation, but how it does this is unknown. It has been suggested that the thiol group (SH) of cysteamine may form a mixed disulphide with the SH groups of tissue proteins, thus protecting the protein $\mathrm{SH}$ groups from attack by free radicals which are formed by ionizing radiation. One objection to this theory is the uncertainty about the extent to which proteins form mixed disulphides with small molecular weight thiols under physiological conditions. Modig (Biochem. Pharmacol., 1\%, 177; 1968) has now shown that normal preparations of proteins do contain mixed disulphides of proteins and small thiols. He has estimated protein and non-protein sulphydryl groups in crystalline bovine serum albumin and in preparations of protein from Ehrlich ascites tumour cells before and after treatment with sodium borohydride to reduce the disulphide bonds. In both preparations there was an increase in both protein and non-protein sulphydryl groups after treatment with sodium borohydride. Usually, three times as much thiol was bound to protein as was bound to other small molecular weight thiols. The author points out that this accounts for discrepancies in earlier estimations of disulphides in proteins because these have sometimes estimated protein disulphides and small disulphides together. 25 per cent of the thiol compounds liberated from the proteins was glutathione, but the other compounds were not characterized.

The protective effect of cysteamine when irradiation is given at different stages of the cell cycle has been studied in tissue cultures of Chinese hamster cells growing synchronously by Sinclair (Science, 159, 442; 1968). Cysteamine reduced the number of cells killed by X-irradiation at all times. The protective effect was greatest, however, in those stages of the cell cycle when the cells are normally very sensitive to radiation, such as the $G_{1}$ period just after mitosis, and least in the stages of the cell cycle when the cells are normally more resistant (the end of the synthetic period $S$ ). When relatively high concentrations of cysteamine were added, however, the number of cells killed did not depend on the stage of the cell cycle at which irradiation took place.

Using the same experimental material-synchronously dividing Chinese hamster cells in tissue cultureKlevecz and Puddle (Science, 159, 634; 1968) have shown that the activity of lactate dehydrogenase and glucose 6-phosphate dehydrogenase increases intermittently during the cell cycle. The increases occur during $G_{1}$ (three hours after mitosis), in the middle of the synthetic period (seven hours after mitosis) and in the late stages of the synthetic period (ten hours after mitosis). The increases correlated with increases in cell size and total protein, suggesting that enzyme synthesis generally varied in this cyclic manner during the cell cycle. This result suggests that cysteamine radioprotection is not due to an effect on protein synthesis, though there may be a difference in the number of proteins with sulphydryl groups synthesized during the sensitive $G_{1}$ phase and the resistant $S$ phase.

\section{Cyclic Antibiotics as Cation Carriers}

\section{from a correspondent}

THE elucidation by Kilbourn et al. (J. Mol. Biol., 30, $559 ; 1967)$ of the molecular structure of a crystalline complex formed between potassium ions and nonactin, one of the cyclic antibiotics, may account for many of the curious properties of this substance and, by inference, for other antibiotics of the same type. These antibiotics (the cyclic polypeptide valinomycin and the gramicidins are more familiar examples) act by allowing potassium ions, protons and other cations but generally not sodium ions, to move across cell membranes and, in particular, across the mitochondrial membrane (Pressman et al., Proc. US Nat. Acad. Sci., $58,1949 ; 1967)$.

A significant feature of their chemical structure is the 